OPEN ACCESS

Edited by:

Christoph Sinning,

University Heart and Vascular Center Hamburg (UHZ), Germany

Reviewed by:

Siva K. Panguluri, University of South Florida,

United States

Bastiaan Geelhoed,

University Medical Center

Groningen, Netherlands

*Correspondence:

Jingyi Ren

renjingyi1213@hotmail.com

Tianbing Wang

wangtianbing@pkuph.edu.cn

tThese authors have contributed equally to this work

Specialty section:

This article was submitted to Cardiovascular Genetics and Systems

Medicine,

a section of the journa

Frontiers in Cardiovascular Medicine

Received: 09 July 2021 Accepted: 28 October 2021 Published: 26 November 2021

Citation:

Gao W, Fan J, Sun D, Yang M, Guo W, Tao L, Zheng J, Zhu J,

Wang $T$ and Ren J (2021) Heart Failure Probability and Early Outcomes of Critically III Patients With COVID-19:

A Prospective, Multicenter Study.

Front. Cardiovasc. Med. 8:738814.

doi: 10.3389/fcvm.2021.738814

\section{Heart Failure Probability and Early Outcomes of Critically III Patients With COVID-19: A Prospective, Multicenter Study}

\author{
Weibo Gao ${ }^{1 \dagger}$, Jiasai Fan ${ }^{2 \dagger}$, Di Sun ${ }^{2 \dagger}$, Mengxi Yang ${ }^{2}$, Wei Guo ${ }^{3}$, Liyuan Tao ${ }^{4}$, \\ Jingang Zheng ${ }^{2}$, Jihong Zhu ${ }^{1}$, Tianbing Wang ${ }^{3 *}$ and Jingyi Ren ${ }^{2 *}$
}

${ }^{1}$ Department of Emergency, Peking University People's Hospital, Beijing, China, ${ }^{2}$ Department of Cardiology, Heart Failure Center, China-Japan Friendship Hospital, Beijing, China, ${ }^{3}$ Trauma Center, Peking University People's Hospital, Beijing, China,

${ }^{4}$ Research Center of Clinical Epidemiology, Peking University Third Hospital, Beijing, China

Background: The relationship between cardiac functions and the fatal outcome of coronavirus disease 2019 (COVID-19) is still largely underestimated. We aim to explore the role of heart failure (HF) and NT-proBNP in the prognosis of critically ill patients with COVID-19 and construct an easy-to-use predictive model using machine learning.

Methods: In this multicenter and prospective study, a total of 1,050 patients with clinical suspicion of COVID-19 were consecutively screened. Finally, 402 laboratory-confirmed critically ill patients with COVID-19 were enrolled. A "triple cut-point" strategy of NTproBNP was applied to assess the probability of HF. The primary outcome was 30-day all-cause in-hospital death. Prognostic risk factors were analyzed using the least absolute shrinkage and selection operator (LASSO) and multivariate logistic regression, further formulating a nomogram to predict mortality.

Results: Within a 30-day follow-up, $27.4 \%$ of the 402 patients died. The mortality rate of patients with HF likely was significantly higher than that of the patient with gray zone and HF unlikely (40.8\% vs. 25 and 16.5\%, respectively, $P<0.001)$. HF likely [Odds ratio (OR) 1.97, 95\% Cl 1.13-3.42], age (OR 1.04, 95\% Cl 1.02-1.06), lymphocyte (OR 0.36, 95\% $\mathrm{Cl} 0.19-0.68$ ), albumin (OR 0.92, 95\% Cl 0.87-0.96), and total bilirubin (OR 1.02, 95\% $\mathrm{Cl} 1-1.04)$ were independently associated with the prognosis of critically ill patients with COVID-19. Moreover, a nomogram was developed by bootstrap validation, and C-index was 0.8 (95\% Cl 0.74-0.86).

Conclusions: This study established a novel nomogram to predict the 30-day allcause mortality of critically ill patients with COVID-19, highlighting the predominant role of the "triple cut-point" strategy of NT-proBNP, which could assist in risk stratification and improve clinical sequelae.

Keywords: COVID-19, heart failure, NT-ProBNP, nomogram, prognosis 


\section{Prevalence}

Non-survivors

Survivors

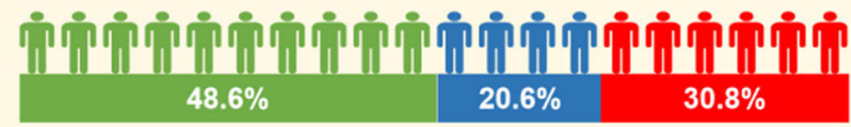

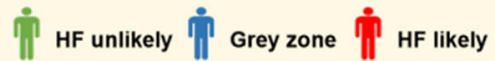

Potential mechanisms

NT-proBNP

(Increased myocardial stress)

SARS-Cov-2
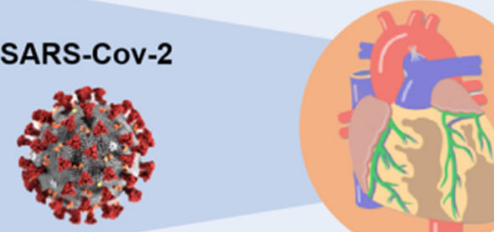

LV Syst. dysfunction

LV Diast. dysfunction

RV dysfunction

PAH

.......
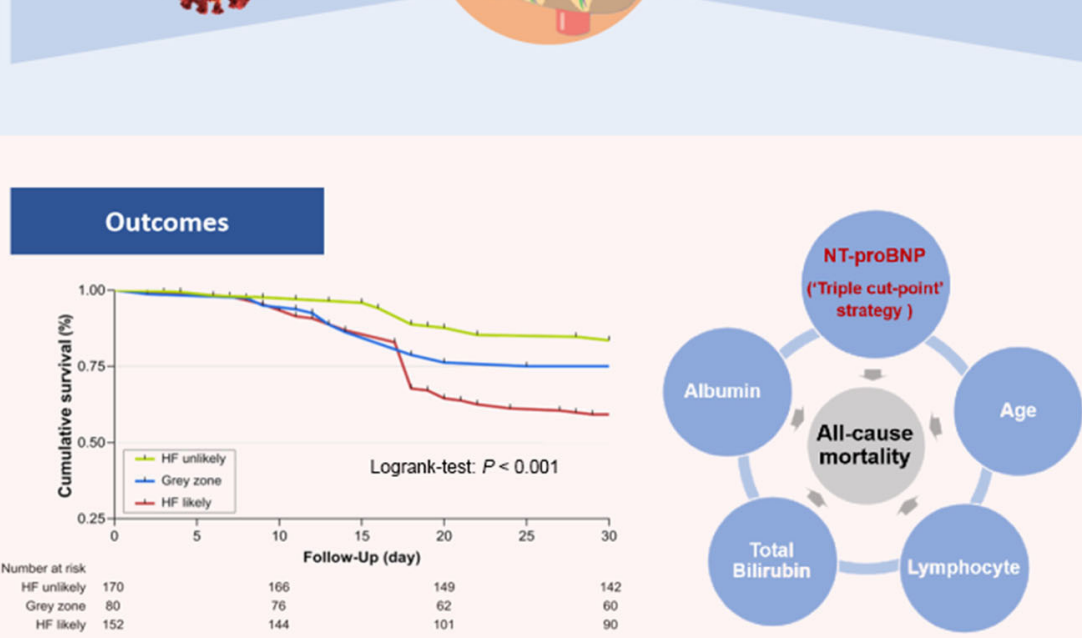

Graphical abstract Heart failure probability and early outcomes of critically ill COVID-19 patients

GRAPHICAL ABSTRACT | Heart failure probability and early outcomes of critically ill COVID-19 patients. HF, heart failure; NT-proBNP, N-terminal probrain natriuretic peptide; LV, left ventricular; RV, right ventricular; PAH, pulmonary arterial hypertension. 


\section{INTRODUCTION}

An outbreak of novel infectious pneumonia, now known as coronavirus disease 2019 (COVID-19) caused by severe acute respiratory syndrome coronavirus 2, has been quickly spreading around the world since December 2019. To date, more than 168 million confirmed cases of COVID-19 have been identified worldwide, with over 3.49 million deaths. Despite the advancement of learning the etiology and clinical characteristics of COVID19, there have been no effective strategies to wipe out the global COVID-19 epidemic, and it is still a public health threat.

The average mortality rate was estimated globally at $3.4 \%$ by the WHO, while it is $26-52 \%$ significantly higher for patients admitted to intensive care units (ICUs) $(1,2)$. Moreover, no medications have been proven definitely effective for curing COVID-19 (3). Thus, early evaluation and identification of individuals with high-risk mortality are of paramount importance to further guide optimal intervention strategies. Of note, critically ill patients with COVID-19 usually have multiple organ dysfunctions (4), among which cardiac involvement is prevalent, especially acute heart failure (AHF) (5). However, the role of AHF in the prognosis of COVID-19 has not been fully elucidated in prior studies, partially because comprehensive evaluations of cardiac dysfunction that utilize imaging examinations were usually unavailable in real-world practice. Hence, we proposed a "triple cut-point" strategy of N-terminal pro-brain natriuretic peptide (NT-proBNP) as a reliable and easy-touse diagnostic tool for AHF in this study (6). Moreover, although previous studies have explored the risk factors of prognosis among critically ill patients with COVID-19 (7-9), a user-friendly and clinically relevant short-time outcome prediction model for patients with COVID-19 in ICU is still lacking.

Therefore, to address the gaps mentioned above, this multicenter study aims to (1) explore the potential prognostic value of the "triple cut-point" strategy of NT-proBNP and AHF in critically ill patients with COVID-19; and (2) construct and validate a simplified and effective nomogram to predict all-cause in-hospital death risk individually.

\section{MATERIALS AND METHODS}

\section{Study Population}

This multicenter, prospective, and observational study consecutively enrolled 1,050 patients with clinical suspicion of COVID-19 from four ICUs in Wuhan taken over by ChinaJapan Friendship Hospital, Peking University People's Hospital, Peking University First Hospital, and Peking University Third Hospital from January to May 2020. Patients who met one of the following criteria would be considered to be transferred to the ICU: (1) respiratory rate $>30$ breath $/ \mathrm{min}$; (2) blood oxygen $\left(\mathrm{SpO}_{2}\right)<93 \%$; (3) $\mathrm{PaO}_{2} / \mathrm{FiO}_{2}<300 \mathrm{mmHg}$; (4) presented with respiratory failure; (5) presented with shock; or (6) other conditions that need to be monitored in the ICU. Patients were diagnosed as COVID-19 with a positive result of real-time reverse transcriptase-polymerase chain reaction assay from nasal swab specimens according to WHO guidance (10). Exclusion criteria included patients who were not diagnosed with COVID-19, younger than 18 years of age, and had incomplete data, or died within $24 \mathrm{~h}$ of admission to the ICU. As a result, 402 patients were included in the final analysis. The study was conducted in accordance with the Declaration of Helsinki, and the protocol was approved by the Ethics Committee of Peking University People's Hospital.

\section{Data Collection}

The clinical data from each patient were recorded by experienced physicians following ICU admission and included demographic features, preexisting comorbidities, symptoms, vital signs, and length of ICU stay. The comorbidities included hypertension, coronary heart disease (CHD), diabetes mellitus (DM), chronic kidney disease (CKD), asthma, chronic obstructive pulmonary disease, chronic bronchitis, transient ischemic attack, ischemic stroke, and hemorrhagic stroke.

All the patients, during hospitalization, were followed up for 30 -days or until discharge or death. The primary outcome was 30-day all-cause death after admission.

\section{Laboratory Measurements}

Laboratory values were collected including complete blood count, high-sensitivity cardiac troponin I (hs-cTNI), NTproBNP, biochemical tests, d-dimer, and procalcitonin (PCT). Complete blood count was measured with a Sysmex XN9000 (Sysmex, Kobe, Japan) automatic hematology analyzer. Coagulation parameters, such as d-dimer, were measured with a Stago STA-R automatic blood coagulation analyzer (Stago, Paris, France). Biochemical tests, namely, alanine aminotransferase (ALT), aspartate aminotransferase (AST), total bilirubin, albumin, blood urea nitrogen (BUN), and creatinine were performed using Roche Cobas 8000 automatic biochemical analyzer (Roche, Rotkreuz, Switzerland). HscTNI was measured with an Abbott ARCHITECT i2000SR chemiluminescence immunoanalyzer (Abbott Laboratories, Illinois, United States). Elevated hs-cTNI was defined as plasma levels of hs-cTNI above the 99th-percentile upper reference limit. NT-proBNP was analyzed with a

TABLE 1 | Classification of patients using the "triple cut-point" strategy of NT-proBNP.

\begin{tabular}{lccc}
\hline Setting & \multicolumn{3}{c}{ Cut-off levels of NT-proBNP $(\mathbf{p g} / \mathbf{m L})$} \\
\cline { 2 - 4 } & Age $<50$ & Age 50-75 & Age $>75$ \\
\hline HF unlikely & $<300$ & $300-900$ & $300-1,800$ \\
Gray zone & $300-450$ & $>900$ & $>1,800$ \\
HF likely & $>450$ & & \\
\hline
\end{tabular}

NT-proBNP, N-terminal pro-B type natriuretic peptide; HF, heart failure. 
Roche Cobas e602 electrochemical luminescence analyzer (Roche, Germany).

\section{“Triple Cut-Point" Strategy of NT-proBNP}

According to the recent guideline for HF, novel NT-proBNP cut-off values have been proposed to assist with AHF diagnosis. Hence, we classified the cases into three groups using this "triple cut-point" strategy of NT-proBNP to define the probability of AHF, as shown in Table 1. In detail, HF likely was defined as plasma NT-proBNP level $>450 \mathrm{pg} / \mathrm{ml}$ in patients below 50 years, $>900 \mathrm{pg} / \mathrm{ml}$ in patients between 50 and 75 years, and $>1,800$ $\mathrm{pg} / \mathrm{ml}$ in patients over 75 years (6). HF unlikely was defined as plasma NT-proBNP level $<300 \mathrm{pg} / \mathrm{ml}$ regardless of age, while the stratified approach of $300 \mathrm{pg} / \mathrm{ml}$ to $450 / 900 / 1,800 \mathrm{pg} / \mathrm{ml}$ for ages $<50 / 50-75 />75$ years were considered as "gray zone."

\section{Statistical Analysis}

Continuous variables were presented as mean \pm SD if normally distributed, and median and interquartile range otherwise. The differences between the two groups were compared by the Student $t$-test and Mann-Whitney $U$ test appropriately. Categorical variables were shown as $\mathrm{n}(\%)$ and compared by $\chi^{2}$ test or Fisher exact test when necessary.

Kaplan-Meier survival estimates were calculated, and the logrank test was performed to compare the groups in terms of survival. The least absolute shrinkage and selection operator (LASSO) method (glmnet package), which is appropriate for

TABLE 2 | Baseline characteristics of the cohort.

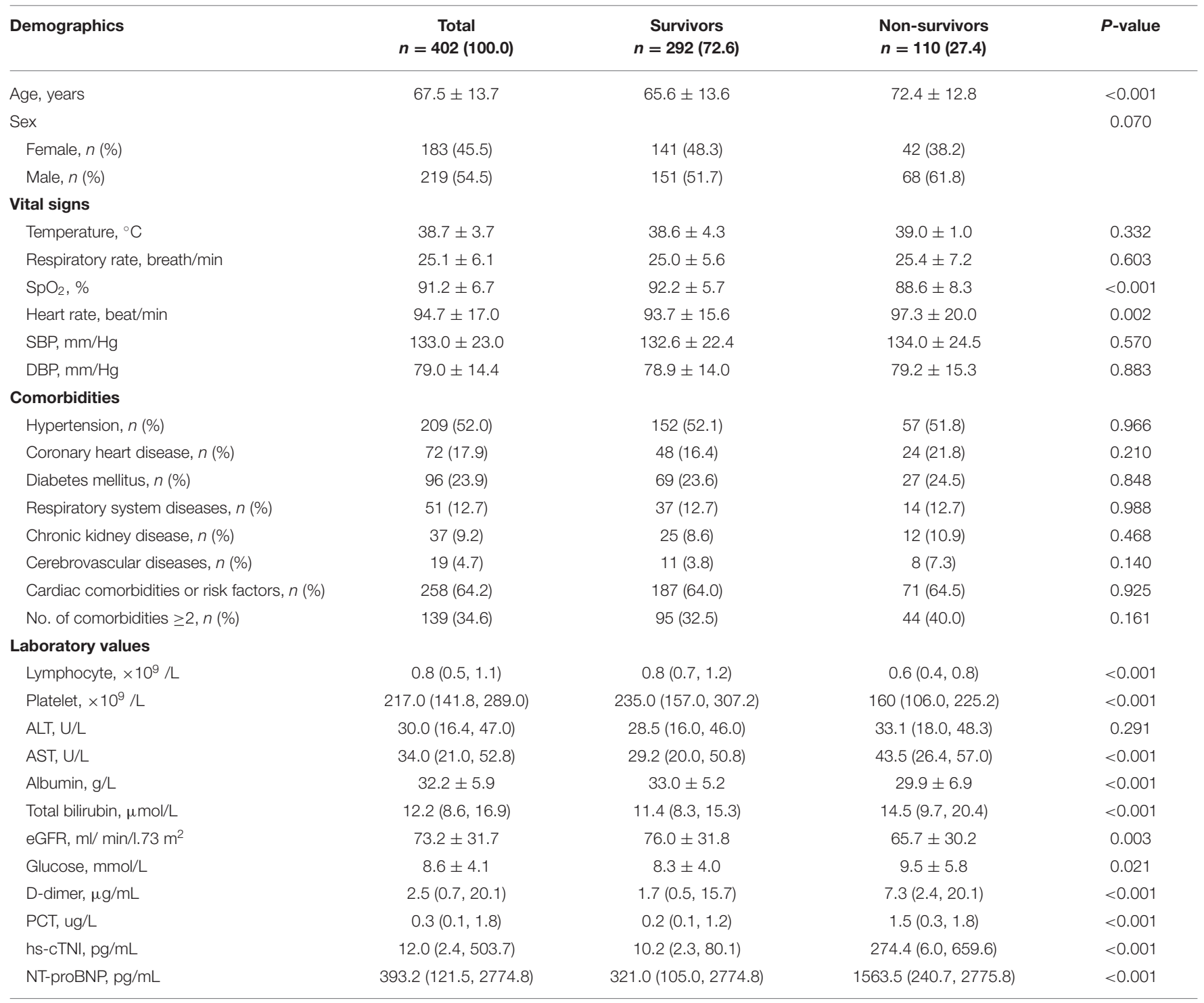

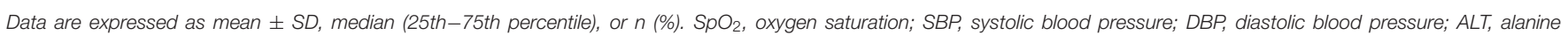

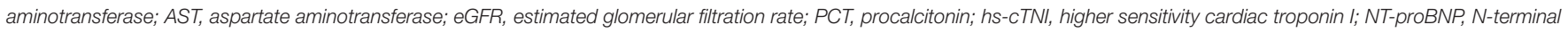
pro-brain natriuretic peptide. 
regression of high-dimensional data, was used to select the most useful predictive variables from the data set. Then, the multivariate logistic regression analysis was performed to identify independent risk factors. Odds ratios (ORs) were shown with a 95\% CI.

The nomogram was established based on the multivariate logistic regression analysis (rms package). A likelihood ratio test approach for model selection was performed. Nomogram performance was quantified with respect to discrimination and calibration. Discrimination (the ability of a nomogram to separate patients with all-cause in-hospital death) was quantified with the concordance index (C-index) and 95\% CI. Calibration was assessed graphically by plotting the relationship between actual (observed) probabilities and predicted probabilities (calibration plot) by Hosmer goodnessof-fit test. The internal validation of performance was estimated with the bootstrapping method (500 replications). Integrated discrimination improvement (IDI) and net reclassification improvement (NRI) (survival package) were used to assess the improved ability of the "triple cut-point" strategy of NT-proBNP for the predictive value of the model.

All the tests were two-tailed, and a $P<0.05$ was considered significant. The statistical analyses were performed with the SPSS version 25.0 software (SPSS Inc., Chicago, IL, United States), R programming language, and environment version 3.6.0 (http:// cran.r-project.org).

\section{RESULTS}

\section{Baseline Characteristics}

This study finally included 402 critically ill patients with laboratory-confirmed COVID-19 and their baseline
A

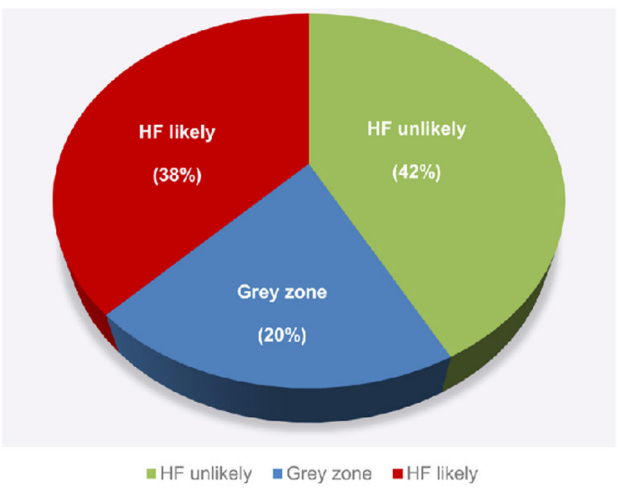

B

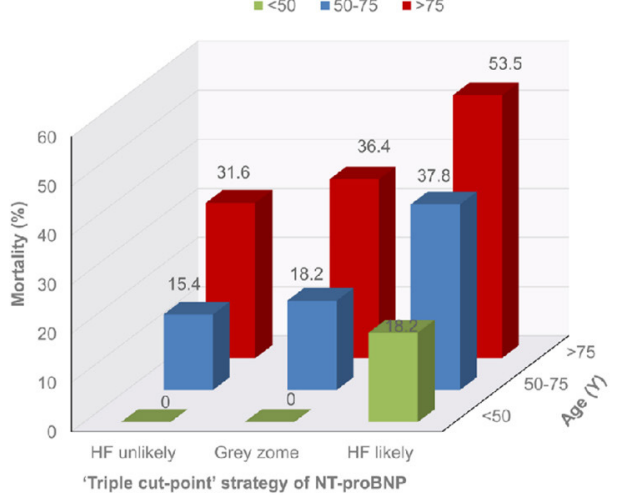

C

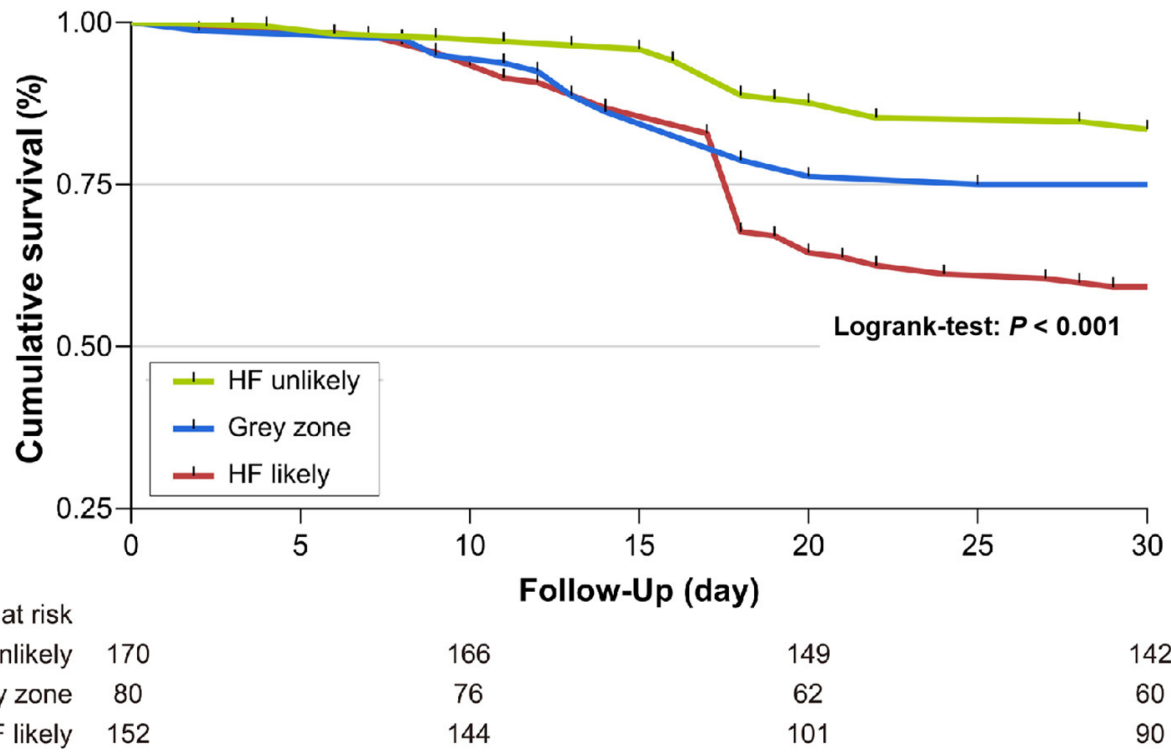

FIGURE 1 | Relationship between the "triple cut-point" strategy of N-terminal pro-brain natriuretic peptide (NT-proBNP) and death. (A) Distribution of the "triple cut-point" strategy of NT-proBNP $(n=402)$. (B) The mortality rate increased with aging and heart failure. (C) Kaplan-Meier survival curves stratified by the "triple cut-point" strategy of NT-proBNP. HF, heart failure. 
characteristics are shown in Table 2. Overall, the mean age of the whole cohort was 67 years, and $54.5 \%(n=219)$ were men. At the 30-day follow-up, 110 patients had died with a $27.4 \%$ mortality risk. Compared to the survivors, the non-survivors were more likely to be older, having decreased $\mathrm{SpO}_{2}$ and elevated heart rate (HR) (all $P<0.05$ ). Of note, there was no significance between the two groups regarding gender and comorbidities, irrespective of hypertension, CHD, DM, and respiratory system disease. Furthermore, we compared the laboratory data between the two groups and found that the nonsurvivors had significantly increased AST, total bilirubin, blood

TABLE 3 | Distribution of "triple cut-point" strategy of NT-proBNP in critically ill patients with coronavirus disease 2019 (COVID-19).

\begin{tabular}{lcccc}
\hline Setting & $\begin{array}{c}\text { Total } \\
\boldsymbol{n}=\mathbf{4 0 2} \\
\mathbf{( 1 0 0 . 0 )}\end{array}$ & $\begin{array}{c}\text { Survivors } \\
\boldsymbol{n}=\mathbf{2 9 2} \\
\mathbf{( 7 2 . 6 )}\end{array}$ & $\begin{array}{c}\text { Non- } \\
\text { survivors } \\
\boldsymbol{n}=\mathbf{1 1 0} \\
\mathbf{( 2 7 . 4 )}\end{array}$ & P-value \\
\hline HF unlikely, $n(\%)$ & $170(42.3)$ & $142(48.6)$ & $28(25.5)$ & $<0.001$ \\
Gray zone, $n(\%)$ & $80(19.9)$ & $60(20.6)$ & $20(18.2)$ & \\
HF likely, $n(\%)$ & $152(37.8)$ & $90(30.8)$ & $62(56.3)^{\star}$ & \\
\hline
\end{tabular}

Data are expressed as n (\%). NT-proBNP, N-terminal pro-brain natriuretic peptide; HF, heart failure.

*The distribution of HF likely between survivors and non-survivors was confirmed to be significantly different by post-hoc test $(p<0.001)$. glucose, d-dimer, and PCT as well as decreased lymphocytes, platelets, and estimated glomerular filtration rate (eGFR) (all $P<0.05)$

\section{"Triple Cut-Point" Strategy of NT-proBNP and 30-Day Mortality}

According to the "triple cut-point" strategy of NT-proBNP, the patients were divided into three groups, and the overall distribution of HF unlikely, gray zone, and HF likely was 170 (42.3\%), 80 (19.9\%), and 152 (37.8\%), respectively (Figure 1A). As shown in Table 3, the non-survivor group has a significantly higher percentage of patients with HF likely (56.3 vs. $30.8 \%$ ), and the distribution of the three groups (HF unlikely, gray zone, and HF likely) between the non-survivor and survivor groups was significantly different $(P<0.001)$. Otherwise, within the 30-day follow-up, we observed a mortality rate of $16.5(28 / 170), 25(20 / 80)$, and $40.8 \%(62 / 152)$ in group HF unlikely, gray zone, and HF likely, respectively $(P<0.001)$. Importantly, the mortality rate increased sharply, accompanied by the increased likelihood of AHF (Figure 1B). The KaplanMeier curves of short-time survival were shown in the central illustration, illustrating a significantly shorter mean survival time for patients with HF likely (Figure 1C). The overall cumulative risk of death at 30-days was significantly higher for the HF likely group than for HF unlikely and gray zone $(P<0.001)$.

\section{A} $\begin{array}{lllllllllllllllll}28 & 28 & 28 & 28 & 28 & 28 & 28 & 27 & 26 & 23 & 16 & 10 & 9 & 7 & 7 & 5 & 2\end{array}$

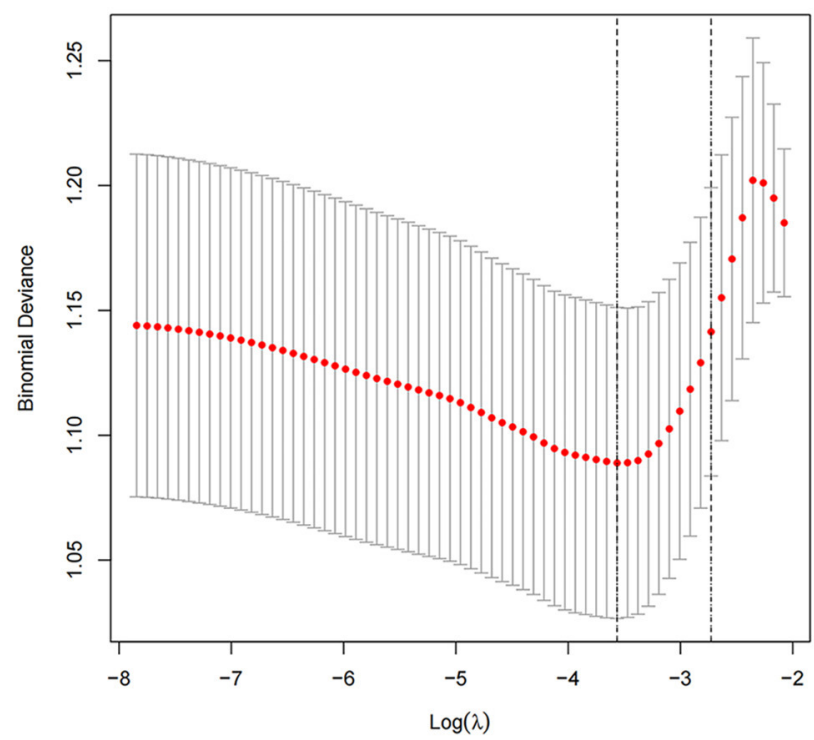

B

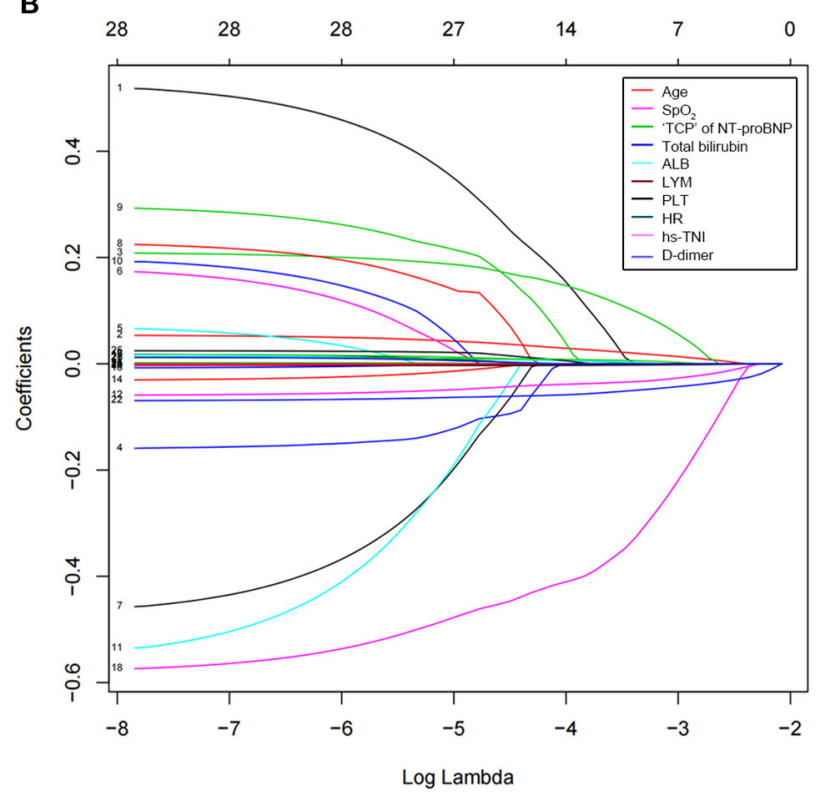

FIGURE 2 | Clinical feature selection using a least absolute shrinkage and selection operator (LASSO) binary logistic regression model. (A) Selection of optimal parameters (lambda) from the LASSO model using 10-fold cross-validation and minimum criteria. The partial likelihood deviance (binomial deviance) curve was plotted vs. log (lambda). Dotted vertical lines were drawn at the optimal values using the minimum criteria and the 1 standard error of the minimum criteria (1-SE criteria). (B) LASSO coefficient profiles of the 28 texture features. A vertical line was drawn at the value selected using 10-fold cross-validation, where optimal I resulted in nine non-zero coefficients. LASSO, least absolute shrinkage and selection operator; hs-cTNI, higher sensitivity cardiac troponin I; "TCP" of NT-proBNP, "triple cut-point" strategy of NT-proBNP; $\mathrm{SpO}_{2}$, blood oxygen; HR, heart rate. 
TABLE 4 | Multivariate logistic regression analyses of risk factors for 30-day mortality.

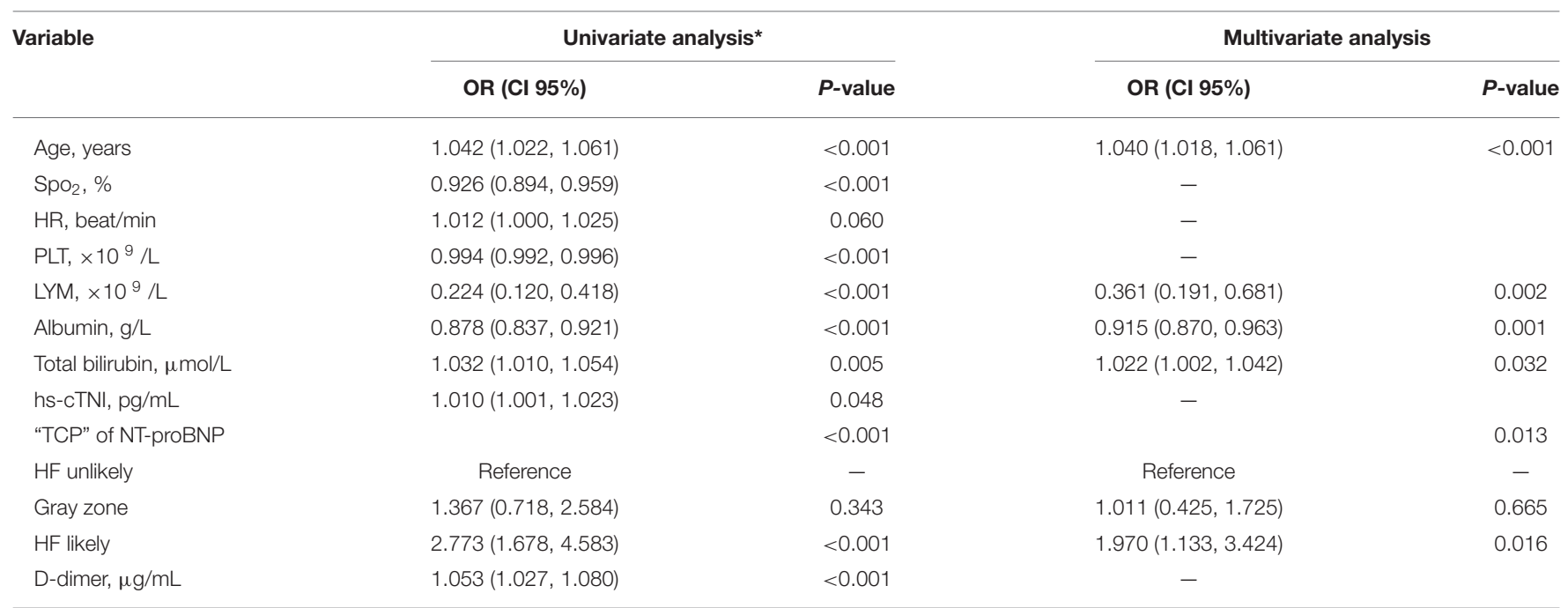

*The variables of the univariate analysis were from the least absolute shrinkage and selection operator (LASSO) binary logistic regression model. $\mathrm{SpO}_{2}$, blood oxygen; HR, heart rate; hs-cTNI, higher sensitivity cardiac troponin l; "TCP" of NT-proBNP, "triple cut-point" strategy of NT-proBNP.

\section{Predictors of 30-Day in-hospital Death of Critically III Patients With COVID-19}

The least absolute shrinkage and selection operator was used to select the potential prognostic factors from numerous parameters. Finally, 28 indexes were reduced to 10 potential predictors, namely, age, lymphocyte, platelet, total bilirubin, "triple cut-point" strategy of NT-proBNP, $\mathrm{SpO}_{2}, \mathrm{HR}$, albumin, hs-cTNI, and d-dimer, based on the 402 patients, and were indexes with non-zero coefficients in the LASSO regression model (Figures 2A,B). Furthermore, as shown in Table 4, the multivariate logistic regression analysis displays five independent predictors for the short-time fatal outcome, namely, HF likely (OR 1.97, 95\% CI 1.133-3.424), older age (OR 1.04, 95\% CI $1.018-1.061$ ), lymphocyte (OR $0.361,95 \%$ CI $0.191-0.681$ ), total bilirubin (OR 1.022, 95\% CI 1.002-1.042), and albumin (OR $0.915,95 \%$ CI $0.87-0.963$ ) (all $P<0.05$ ).

\section{Development and Validation of a Novel Nomogram for Predicting Prognosis}

An optimal nomogram comprising all the above independent predictors was established to individualize the risk of 30-day inhospital death (Figure 3A). The ratios of calculated $\beta$ were used to decide the proportional prognostic effect of these variables. Projections from total points on the scales below indicated the estimated probability of death.

Performance accuracy was evaluated by the area under the curve (AUC) of the receiver operating characteristic (ROC) analysis. The AUC for in-hospital death was 0.781 (95\% CI $0.733-$ 0.827 ) (Figure 3B). The calibration curve of the nomogram for the probability of death demonstrated good agreement between prediction and observation in the primary cohort (Figure 3C). Hosmer-Lemeshow goodness-of-fit was satisfied $(P=0.354)$. The C-index for the prediction nomogram was 0.798 (95\% CI $0.742-0.857$ ). The decision curve analysis (DCA) for the clinical laboratory index nomogram is presented in Figure 3D. It showed that this nomogram had more benefits than the treat-all-patients scheme or the treat-none scheme in predicting the risk of 30day in-hospital death of critically ill patients with COVID-19. Moreover, the bootstrap validation method was used to verify the predictive accuracy of the nomogram. The C-index for the nomogram of 30-day in-hospital death was 0.779 (95\% CI 0.721 0.834 ), suggesting the accuracy of this predictive nomogram.

\section{Incremental Predictive Value of "Triple Cut-Point" Strategy of NT-proBNP}

To investigate the role of the "triple cut-point" strategy of NTproBNP in the predictive value of the current model, NRI and IDI were calculated. Compared with the model without the "triple cut-point" strategy of NT-proBNP, the addition of the "triple cut-point" strategy of NT-proBNP resulted in a significantly improved discrimination [IDI 7.3\% (95\% CI 1.1-14.5\%) and NRI 4.9\% (95\% CI 2.6-7.2\%), both with $P<0.05$ ].

\section{DISCUSSION}

In this prospective multicenter study, we recruited 402 critically ill patients with COVID-19 from four ICUs in China and established a novel nomogram to predict the 30-day all-cause mortality risk in these patients. To the best of our knowledge, there have been few risks score models for predicting the prognosis of critically ill patients with COVID-19. This study has developed a user-friendly and relatively personalized model incorporating five variables, age, "triple cut-point" strategy of NT-proBNP, albumin, lymphocyte count, and total bilirubin, to predict short-time mortality risk in critically ill Chinese patients with COVID-19, which could assist risk stratification and provide insights for timely interventions upon admission. Furthermore, it is highlighted that the "triple cut-point" strategy of NT-proBNP 


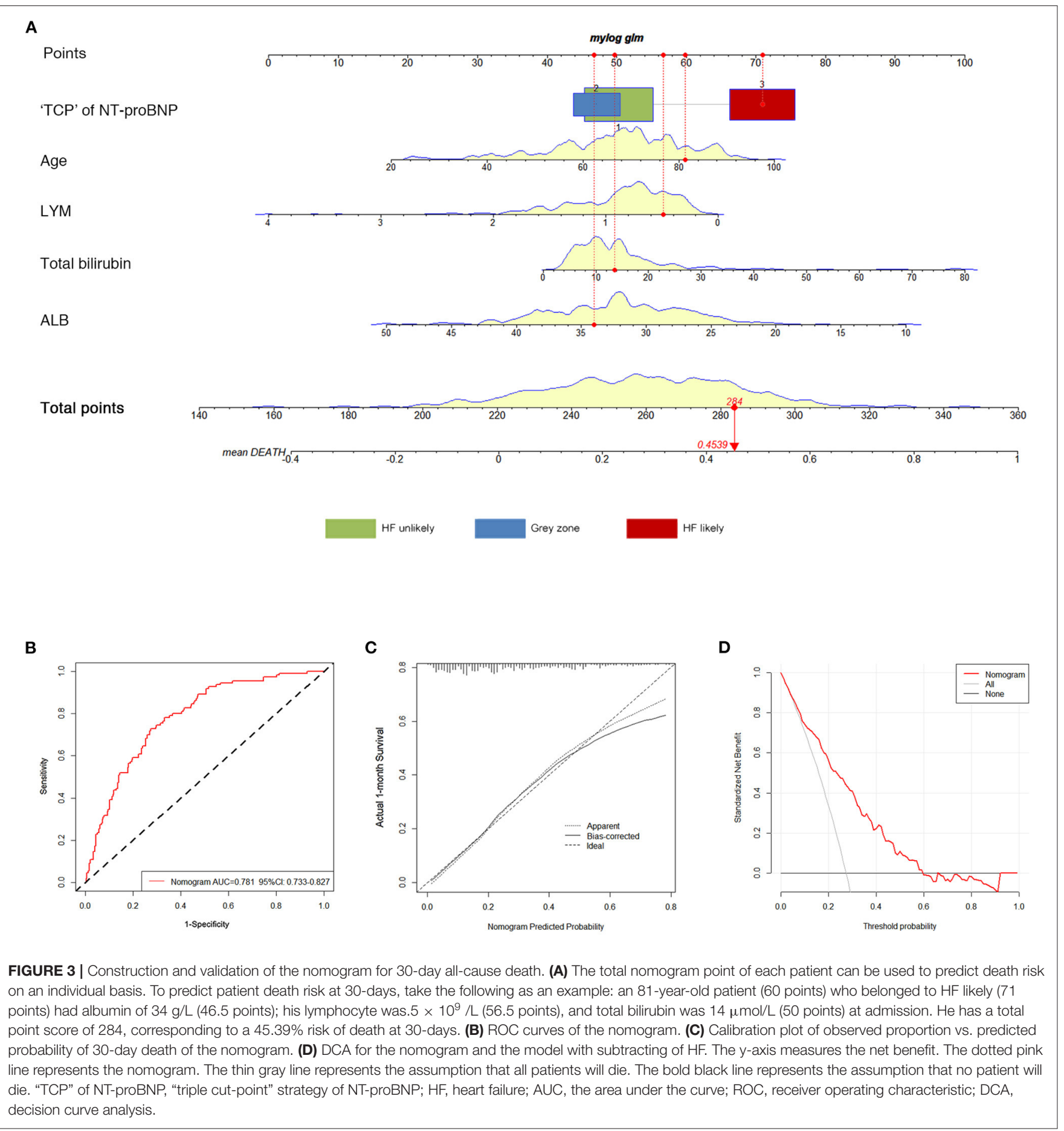

demonstrated the predominant role of AHF in the clinical course and prognosis in COVID-19.

The mortality risk of COVID-19 has been proven high, with 28 -day mortality ranging from $26-53.8 \%$ in critically ill adult patients worldwide $(1,11)$, indicating the imperative of proposing an easy-to-use prediction model to assist riskstratify and therapeutic optimization in clinical practice for ICU patients. Emerging evidence has tried to explore the risk factors and construct diagnostic and prognostic models in COVID-19 populations $(12,13)$. However, previous reports regarding prognosis prediction have mainly focused on disease progression or mortality risk of the whole group without further distinguishing critically ill patients in ICU (14). The sample selection bias of the prior models could lead to poor adaptions. In line with existing data, this study reported a $27.6 \%$ mortality risk, and further constructed and validated a novel nomogram 
for the prediction of 30-day all-cause death. Variables referring to older age, higher level of total bilirubin, lower level of lymphocyte count and albumin, and "triple cut-point" strategy of NT-proBNP were likely to recognize individuals who are at high risk with high sensitivity and specificity. More importantly, the quantitative appraisal made it possible to estimate the likelihood of death more accurately and individually with easy and rapid access in clinical practice.

It has been widely confirmed that cardiac involvement, such as cardiac injury, arrhythmias, myocarditis, and cardiac dysfunction, was prevalent and prognostic in hospitalized patients with COVID-19 (15-17), among which $\mathrm{HF}$ is responsible for substantial morbidity and mortality (10). New-onset HF was observed in nearly $23 \%$ of hospitalized patients with COVID-19 and as much as one-third of those admitted to the ICU $(2,9)$. Recent reports revealed that HF was the most frequent cause of death just after acute respiratory distress syndrome (ARDS) and sepsis, accounting for $27.4 \%$ of the proximate causes of death in patients with COVID-19 (18). However, the role of HF in the prognosis of critically ill patients with COVID-19 has not been fully elucidated, partially because of high diagnostic uncertainty. A complete diagnosis of HF usually includes symptoms, signs, biomarkers (BNP/NTproBNP), and imaging examinations, while it is impractical and unavailable to evaluate cardiac function by echocardiography for each critically ill patient with COVID-19 in the clinical practice. Although BNP/NT-proBNP levels are easily interfered with and obscured by considerable factors, the utility of these biomarkers performed well in the emergency setting as an adjunct tool for the diagnosis and triage of dyspneic patients. As such, the guidance has recommended BNP/NT-proBNP as a diagnostic aid for HF with comparable diagnostic accuracy.

As the widely admitted biomarker in HF, NT-proBNP quantitatively reflects hemodynamic myocardial stress (19), indicating not only left ventricular (LV) systolic dysfunction but also cardiac abnormalities, such as LV diastolic dysfunction, right ventricular (RV) dysfunction, valvular dysfunction, increased pulmonary pressures, and atrial arrhythmias. Prior studies have observed ambiguous results that higher levels of BNP or NTproBNP were found in patients with severe COVID-19 and that they were independently associated with high mortality, maybe because of single-center design, patient population selection bias, and small sample size (20-23). This multicenter study demonstrated that the non-survivors had a significantly higher level of NT-proBNP than the survivors $(1,564 \mathrm{vs} .321 \mathrm{ng} / \mathrm{ml})$, with reasonable sample size. Consistently, a recent study described the characterization of NT-proBNP in patients with COVID-19, and $48.5 \%$ of their cohort presented NT-proBNP levels above the recommended cut-off for the identification of HF (24).

Furthermore, considering the fact that the plasma level of NT-proBNP is largely affected by age and renal functions, it seems to be not rigorous enough to use NT-proBNP as a simple continuous variable alone to predict the prognosis of patients with COVID-19. Thus, we reclassified the subjects into three groups (HF likely, gray zone, and HF unlikely) according to the recent $\mathrm{HF}$ guidance as to the "triple cut-point" strategy of
NT-proBNP, and observed that patients with HF likely occupied $37.8 \%$ of the total cohort, of which $56.3 \%$ were non-survivors (6). Moreover, we found that patients in the HF likely group had a significantly higher risk (OR 1.97, 95\% CI 1.133-3.424) for 30day all-cause death. Concerning the clinical presentations and biomarkers of HF on time would help make optimal individual treatment plans to prevent further deterioration efficiently.

It is worth noting that our prediction model did not incorporate troponin, as it was not independently associated with the outcome unexpectedly, while prior studies have suggested that troponin was a significant prognostic indicator in COVID19 (15, 25). Similarly, Dong et al. conducted a retrospective study and built a nomogram assessing the 14-day and 21-day inhospital survival of all the general patients with COVID-19. The final model was constructed based on hypertension, neutrophilto-lymphocyte ratio, and NT-proBNP (26). Elevated troponin may be a possible confounder for NT-proBNP as they were postulated to share the same pathophysiological processes and found to be both elevated in pneumonia, sepsis, ARDS, and several other non-cardiac illnesses. Hence, we speculated that an accurate classification using the "triple cut-point" strategy of NT-proBNP may decrease the confounding effect of troponin. Notably, liver injuries, such as elevated total bilirubin and decreased albumin, have also been demonstrated to be common and associated with disease severity and poor outcomes for critically ill patients in this study, in accordance with previous studies (27). Furthermore, elevated total bilirubin may also be associated with cardiac dysfunctions as a significant and independent predictor of poor cardiovascular prognosis in patients with HF (28).

Critically ill conditions with COVID-19 were usually complicated by multiple organ dysfunctions with complex pathophysiological processes involving numerous parameters, including but not limited to hypoxemia, inflammation, thromboembolism, renal failure, and cardiac damage (29). Therefore, it is of vital importance to bring all reasonable possible variables into analysis and construct a scientific prediction model relying on appropriate statistical analysis awfully. In the current study, LASSO, a machine learning algorithm, was applied to shrink the regression coefficients from amounting clinical and laboratory indicators to 10 potential predictors. Thus, this algorithm could conquer common confusing collinearity issues and yield more robust results than traditional variable screening methods.

Some cautions should be considered when interpreting our results. First, although our study is observational and the sample size is relatively small, it has a multicenter and prospective design emphasizing critically ill patients. Further investigations with a larger sample size are warranted. Second, this study did not apply other diagnostic tools to make a complete $\mathrm{HF}$ diagnosis. However, it is impractical and unavailable to evaluate cardiac functions by echocardiography for each critically ill patient with COVID-19. Conversely, rapid measurements of NT-proBNP have substantial medical aids to fulfill the clinical need underlying this extraordinary stressful setting, although it should never be a stand-alone test for HF diagnosis. Third, our nomogram model lacks validation in an external 
population. Nevertheless, internal verification indicated the predictive strength in our study.

\section{CONCLUSIONS}

In this study, we explored the independent predictors for shorttime prognosis in critically ill patients with COVID-19 in China and established a novel nomogram to predict the 30-day all-cause mortality risk for the first time, highlighting the predominant role of the "triple cut-point" strategy of NT-proBNP. This easy-touse prognostication nomogram can provide survival estimations and help identify patients with COVID-19 with a high-risk trajectory, further advancing clinical management and ultimately improving outcomes.

\section{DATA AVAILABILITY STATEMENT}

The raw data supporting the conclusions of this article will be made available by the authors, without undue reservation.

\section{ETHICS STATEMENT}

The studies involving human participants were reviewed and approved by the Ethics Committee of Peking University People's Hospital. The ethics committee waived the requirement for written informed consent.

\section{AUTHOR CONTRIBUTIONS}

JR and TW conceived and designed the study and coordinated to complete the study. JR critically revised the manuscript.

\section{REFERENCES}

1. Grasselli G, Zangrillo A, Zanella A, Antonelli M, Cabrini L, Castelli A, et al. Baseline characteristics and outcomes of 1591 patients infected with SARS-CoV-2 admitted to ICUs of the Lombardy Region, Italy. JAMA. (2020) 323:1574-81. doi: 10.1001/jama.2020.5394

2. Arentz M, Yim E, Klaff L, Lokhandwala S, Riedo FX, Chong M, et al. Characteristics and outcomes of 21 critically ill patients with COVID-19 in Washington state. JAMA. (2020) 323:1612-4. doi: 10.1001/jama.2020.4326

3. Casadevall A, Joyner MJ, Pirofski L-A. A Randomized trial of convalescent plasma for COVID-19-potentially hopeful signals. JAMA. (2020) 324:455-7. doi: 10.1001/jama.2020.10218

4. Wang T, Du Z, Zhu F, Cao Z, An Y, Gao Y, et al. Comorbidities and multi-organ injuries in the treatment of COVID-19. Lancet. (2020) 395:e52. doi: 10.1016/S0140-6736(20)30558-4

5. Aboughdir M, Kirwin T, Abdul Khader A, Wang B. Prognostic value of cardiovascular biomarkers in COVID-19: a review. Viruses. (2020) 12:527. doi: $10.3390 / \mathrm{v} 12050527$

6. Mueller C, McDonald K, de Boer RA, Maisel A, Cleland JGF, Kozhuharov N, et al. Heart Failure Association of the European Society of Cardiology practical guidance on the use of natriuretic peptide concentrations. Eur J Heart Fail. (2019) 21:715-31. doi: 10.1002/ejhf.1494

7. Yang X, Yu Y, Xu J, Shu H, Xia J, Liu H, et al. Clinical course and outcomes of critically ill patients with SARS-CoV-2 pneumonia in Wuhan, China: a single-centered, retrospective, observational study. Lancet Respir Med. (2020) 8:475-81. doi: 10.1016/S2213-2600(20)3 0079-5
WG contributed to data collection and completed the project. JF and DS analyzed and interpreted the data and wrote the manuscript. LT assisted in performing statistical analysis. MY, WG, JZhe, and JZhu helped revise the manuscript for important intellectual content and language polishing. All the authors have read and approved the final version of the manuscript.

\section{FUNDING}

This study was supported by the National Natural Science Foundation of China (81770359 to JR), State Key Laboratory of Molecular Developmental Biology of China (2020-MDB-KF-17 to JR), Beijing Health Science and Technology Achievements and Appropriate Technology Promotion Project (BHTPP202004 to JR), Elite Medical Professionals project of China-Japan Friendship Hospital (ZRJY2021-BJ01 to JR), and China-Japan Friendship Hospital Scientific Research Funds (2019-2-QN-77 to DS).

\section{ACKNOWLEDGMENTS}

We sincerely acknowledge the healthcare workers from Zhongfaxincheng campus and Guanggu campus of Tongji Hospital Affiliated with Huazhong University of Science and Technology in Wuhan. They contributed tremendously to blocking the spread and prevalence of COVID-19 in Wuhan, Hubei Province, China, and the whole country.

8. Yu Y, Xu D, Fu SZ, Zhang J, Yang XB, Xu L, et al. Patients with COVID-19 in 19 ICUs in Wuhan, China: a cross-sectional study. Crit Care. (2020) 24:219-28. doi: 10.1186/s13054-020-02939-x

9. Haase N, Plovsing R, Christensen S, Poulsen LM, Brøchner AC, Rasmussen $\mathrm{BS}$, et al. Characteristics, interventions, and longer term outcomes of COVID19 ICU patients in Denmark-a nationwide, observational study. Acta Anaesthesiol Scand. (2021) 65:68-75. doi: 10.1111/aas.13701

10. WHO. Clinical Management of Severe Acute Respiratory Infection When Novel Coronavirus ( $\mathrm{nCoV}$ ) Infection Is Suspected: Interim Guidance, 25 January 2020 (2020).

11. Xie J, Wu W, Li S, Hu Y, Hu M, Li J, et al. Clinical characteristics and outcomes of critically ill patients with novel coronavirus infectious disease (COVID19) in China: a retrospective multicenter study. Intensive CareMed. (2020) 46:1863-72. doi: 10.1007/s00134-020-06211-2

12. Feng Z, Yu Q, Yao S, Luo L, Zhou W, Mao X, et al. Early prediction of disease progression in COVID-19 pneumonia patients with chest CT and clinical characteristics. Nat Commun. (2020) 11:4968-76. doi: $10.1038 / \mathrm{s} 41467-020-18786-\mathrm{x}$

13. Wynants L, Van Calster B, Collins GS, Riley RD, Heinze G, Schuit E, et al. Prediction models for diagnosis and prognosis of covid-19: systematic review and critical appraisal. BMJ. (2020) 369:m1328. doi: 10.1136/bmj. $\mathrm{m} 1328$

14. Vultaggio A, Vivarelli E, Virgili G, Lucenteforte E, Bartoloni A, Nozzoli C, et al. Prompt predicting of early clinical deterioration of moderate-tosevere COVID-19 patients: usefulness of a combined score using IL-6 in a preliminary study. J Allergy Clin Immunol Pract. (2020) 8:2575-81.e2. doi: 10.1016/j.jaip.2020.06.013 
15. Inciardi RM, Adamo M, Lupi L, Cani DS, Di Pasquale M, Tomasoni D, et al. Characteristics and outcomes of patients hospitalized for COVID19 and cardiac disease in Northern Italy. Eur Heart J. (2020) 41:1821-9. doi: 10.1093/eurheartj/ehaa388

16. Wang D, Hu B, Hu C, Zhu F, Liu X, Zhang J, et al. Clinical characteristics of 138 hospitalized patients with 2019 novel coronavirus-infected pneumonia in Wuhan, China. JAMA. (2020) 323:1061-9. doi: 10.1001/jama.2020.1585

17. Liu PP, Blet A, Smyth D, Li H. The science underlying COVID-19: implications for the cardiovascular system. Circulation. (2020) 142:68-78. doi: 10.1161/CIRCULATIONAHA.120.047549

18. Zhang S, Guo M, Duan L, Wu F, Hu G, Wang Z, et al. Development and validation of a risk factor-based system to predict short-term survival in adult hospitalized patients with COVID-19: a multicenter, retrospective, cohort study. Crit Care. (2020) 24:438-50. doi: 10.1186/s13054-020-03123-x

19. Ibrahim NE, Burnett JC, Butler J, Camacho A, Felker GM, Fiuzat M, et al. Natriuretic peptides as inclusion criteria in clinical trials. JACC Heart Fail. (2020) 8:347-58. doi: 10.1016/j.jchf.2019.12.010

20. Gao L, Jiang D, Wen X, Cheng X, Sun M, He B, et al. Prognostic value of NT-proBNP in patients with severe COVID-19. Respir Res. (2020) 21:83-9. doi: 10.1186/s12931-020-01352-w

21. Pranata R, Huang I, Lukito AA, Raharjo SB. Elevated N-terminal pro-brain natriuretic peptide is associated with increased mortality in patients with COVID-19: systematic review and meta-analysis. Postgrad Med J. (2020) 96:387-91. doi: 10.1136/postgradmedj-2020-137884

22. Sorrentino S, Cacia M, Leo I, Polimeni A, Sabatino J, Spaccarotella CAM, et al. B-type natriuretic peptide as biomarker of COVID-19 disease severity-a meta-analysis. J Clin Med. (2020) 9:2957-63. doi: 10.3390/jcm9092957

23. Shi S, Qin M, Shen B, Cai Y, Liu T, Yang F, et al. Association of cardiac injury with mortality in hospitalized patients with COVID-19 in Wuhan, China. JAMA Cardiol. (2020) 5:802-10. doi: 10.1001/jamacardio.2020.0950

24. Caro-Codón J, Rey JR, Buño A, Iniesta AM, Rosillo SO, Castrejon-Castrejon S, et al. Characterization of NT-proBNP in a large cohort of COVID-19 patients. Eur J Heart Fail (2021) 23:456-64. doi: 10.1002/ejhf.2095

25. Guo T, Fan Y, Chen M, Wu X, Zhang L, He T, et al. Cardiovascular implications of fatal outcomes of patients with Coronavirus
Disease 2019 (COVID-19). JAMA Cardiol. (2020) 5:811-18. doi: 10.1001/jamacardio.2020.1017

26. Dong YM, Sun J, Li YX, Chen Q, Liu QQ, Sun Z, et al. Development and validation of a nomogram for assessing survival in patients with COVID-19 pneumonia. Clin Infect Dis. (2020) 72:652-60. doi: 10.1093/cid/ciaa963

27. Bloom PP, Meyerowitz EA, Reinus Z, Daidone M, Gustafson J, Kim AY, et al. Liver biochemistries in hospitalized patients with COVID-19. Hepatology. (2021) 73:890-900. doi: 10.1002/hep.31326

28. Suzuki K, Claggett B, Minamisawa M, Packer M, Zile MR, Rouleau J, et al. Liver function and prognosis, and influence of sacubitril/valsartan in patients with heart failure with reduced ejection fraction. Eur J Heart Fail. (2020) 22:1662-71. doi: 10.1002/ejhf.1853

29. Gupta S, Hayek SS, Wang W, Chan L, Mathews KS, Melamed ML, et al. Factors associated with death in critically ill patients with coronavirus disease 2019 in the US. JAMA Intern Med. (2020) 180:1-12. doi: 10.1001/jamainternmed.2020.3596

Conflict of Interest: The authors declare that the research was conducted in the absence of any commercial or financial relationships that could be construed as a potential conflict of interest.

Publisher's Note: All claims expressed in this article are solely those of the authors and do not necessarily represent those of their affiliated organizations, or those of the publisher, the editors and the reviewers. Any product that may be evaluated in this article, or claim that may be made by its manufacturer, is not guaranteed or endorsed by the publisher.

Copyright (c) 2021 Gao, Fan, Sun, Yang, Guo, Tao, Zheng, Zhu, Wang and Ren. This is an open-access article distributed under the terms of the Creative Commons Attribution License (CC BY). The use, distribution or reproduction in other forums is permitted, provided the original author(s) and the copyright owner(s) are credited and that the original publication in this journal is cited, in accordance with accepted academic practice. No use, distribution or reproduction is permitted which does not comply with these terms. 